\title{
Técnica bulk-fill comparada con técnica incremental para restauraciones posteriores en pacientes con dentición permanente.
}

\section{Bulk-fill technique versus conventional resin composite for posterior restorations in permanent teeth.}

\begin{abstract}
Begoña Larraechea ${ }^{1,2}$, Sara Rodríguez ${ }^{1,2}$, Javier Toledo ${ }^{1,2^{*}}$
1. Escuela de Odontología, Facultad de Medicina, Pontificia Universidad Católica de Chile, Santiago, Chile

2. Proyecto Epistemonikos, Santiago, Chile

* Correspondencia Autor: Javier Toledo | Dirección: Centro Evidencia UC, Pontificia Universidad

Católica de Chile, Diagonal Paraguay 476,

Santiago, Chile | E-mail: Jitoledos@uc.cl

RESUMEN

Introducción: Las restauraciones en el sector posterior son generalmente manejadas mediante el uso de resinas convencionales con técnica incremental. Debido a diversas limitaciones de la técnica convencional, la técnica bulk-fill ha ganado relevancia en la práctica clínica. Este resumen tiene como objetivo evaluar la efectividad de ambas técnicas al momento de realizar restauraciones clase I y clase II de Black en dientes posteriores permanentes. Métodos: Realizamos una búsqueda en Epistemonikos, la mayor base de datos de revisiones sistemáticas en salud, la cual es mantenida mediante el cribado de múltiples fuentes de información, incluyendo MEDLINE, EMBASE, Cochrane, entre otras. Extrajimos los datos desde las revisiones identificadas, analizamos los datos de los estudios primarios, realizamos un metanálisis y preparamos una tabla de resumen de los resultados utilizando el método GRADE. Resultados y conclusiones: Identificamos cinco revisiones sistemáticas que en conjunto incluyeron 15 estudios primarios, todos correspondientes a ensayos aleatorizados. Concluimos que la técnica bulk-fill podría aumentar levemente el fracaso de la restauración a mediano plazo, podría resultar en poca o nula diferencia en el fracaso de la restauración a largo plazo y podría resultar en poca o nula diferencia en el desarrollo de caries secundaria, pero la certeza de la evidencia es baja. La técnica bulk-fill probablemente resulta en poca o nula diferencia en el riesgo de sensibilidad postoperatoria.
\end{abstract}

\section{ABSTRACT}

Introduction: Conventional resin composite has been widely used in the restoration of posterior teeth. However, due to its limitations, the bulk-fill technique has been adopted by clinicians. This evidence summary aims to evaluate the effectiveness of both techniques for class I and II restorations in permanent posterior teeth. Methods: We searched in Epistemonikos, the largest database of systematic reviews in health, which is maintained by screening multiple information sources, including MEDLINE, EMBASE, Cochrane, among others. We extracted data from the systematic reviews, reanalyzed data of primary studies, conducted a meta-analysis and generated a summary of findings table using the GRADE approach. Results and conclusions: We identified five systematic reviews including fifteen studies overall, of which all were randomized trials. We conclude that bulk-fill technique may slightly improve the risk of medium-term restoration failure, may make little or no difference to longterm restoration failure and may make little or no difference to secondary caries but the certainty of the evidence has been assessed as low. Finally, bulk-fill technique probably makes little or no difference to postoperative sensibility.

\section{KEY WORDS}

Bulk-fill technique; Incremental technique; Posterior teeth; Epistemonikos; GRADE.

Int. J. Inter. Dent Vol. 13(3); 196-200, 2020. 


\section{PROBLEMA}

Durante las últimas décadas, diversos materiales han sido utilizados para el tratamiento de caries, fracturas dentarias y pérdidas de tejido dentarios. Los materiales dentales más utilizados son la amalgama y resinas compuestas. Las amalgamas poseen buenas propiedades mecánicas pero su uso ha sido cuestionado por la incorporación de mercurio a la aleación.

En la actualidad, debido a la creciente demanda de restauraciones estéticas, las resinas compuestas han ganado protagonismo en la odontología restauradora ${ }^{[1], ~[2] . ~ E l ~ f r a c a s o ~ c l i ́ n i c o ~ d e ~ e s t a s ~ r e s i n a s ~ e n ~ e l ~}$ tiempo es causado por problemas como fracturas de la restauración, infiltración marginal, sensibilidad postoperatoria y caries secundaria, relacionándose con el estrés de contracción por la polimerización ${ }^{[3]}$. Las resinas convencionales permiten ocupar una técnica incremental, de espesores hasta dos milímetros, disminuyendo la contracción por polimerización, pero aumentando el tiempo clínico de trabajo.

Frente a esto, nuevas tecnologías han surgido como alternativas de técnica restauradora. Una de ellas son las resinas bulk-fill, que permiten una mejor disipación de la luz a través del material y por lo tanto realizar restauraciones directas a través de un solo incremento de mayor espesor (cuatro a cinco milímetros), por lo que disminuye los tiempos de trabajo clínico.

No obstante, se ha planteado que no existe diferencias significativas entre ocupar una técnica incremental o una bulk-fill para dientes posteriores permanentes ${ }^{[4]}$. Este resumen busca evaluar el desempeño clínico del tratamiento entre ambas técnicas.

\section{MÉTODOS}

Realizamos una búsqueda en Epistemonikos, la mayor base de datos de revisiones sistemáticas en salud, la cual es mantenida mediante búsquedas en múltiples fuentes de información, incluyendo MEDLINE, EMBASE, Cochrane, entre otras. Extrajimos los datos desde las revisiones identificadas y analizamos los datos de los estudios primarios. Con esta información, generamos un resumen estructurado denominado FRISBEE (Friendly Summaries of Body of Evidence using Epistemonikos), siguiendo un formato preestablecido, que incluye mensajes clave, un resumen del conjunto de evidencia (presentado como matriz de evidencia en Epistemonikos), metanálisis del total de los estudios cuando sea posible, una tabla de resumen de resultados con el método GRADE y una sección de otras consideraciones para la toma de decisión.

\section{Mensajes clave}

- La técnica bulk-fill podría aumentar levemente el fracaso de la restauración a mediano plazo, podría resultar en poca o nula diferencia en el fracaso de la restauración a largo plazo y en el riesgo de desarrollo de caries secundaria (certeza de la evidencia es baja).

- La técnica bulk-fill probablemente resulta en poca o nula diferencia en la sensibilidad postoperatoria.

\section{Acerca del conjunto de evidencia para esta pregunta}

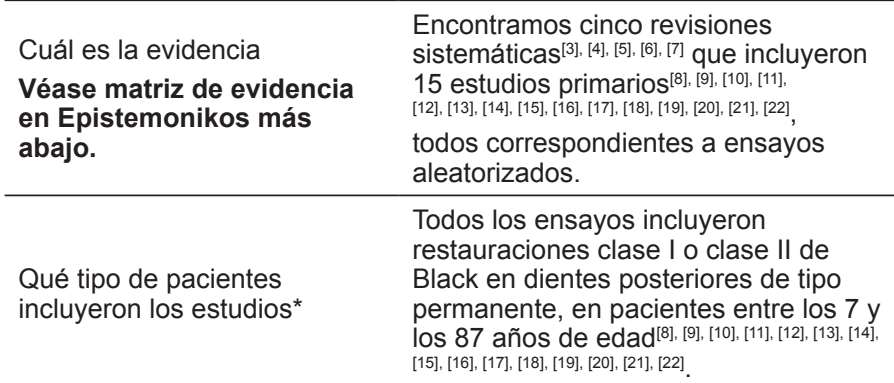

Todos los ensayos compararon el desempeño clínico de restauraciones

Qué tipo de intervenciones incluyeron los estudios* con técnica bulk-fill contra restauración con técnica incrementa[[8], [9], [10], [11], [12], [13], [14], [15], [16], [17], [18], [19], [20], [21], [22]

Los ensayos reportaron múltiples desenlaces, los cuales fueron agrupados por las revisiones sistemáticas de la siguiente manera:

- Fracaso clínico

- Sensibilidad postoperatoria

- Caries secundaria

- Retención o fractura

Qué tipo de desenlaces

- Adaptación marginal midieron

- Decoloración marginal

- Forma anatómica

- Color

- Rugosidad superficial

El seguimiento promedio de los ensayos fue de 38 meses con un rango que fluctúa entre 12 meses y 10 añoS [8], [9], [10], [11], [12], [13], [14], [15], [16], [17], [18], [19] [20], [21], [22].

* La información sobre los estudios primarios es extraída desde las revisiones sistemáticas identificadas, no directamente desde los estudios, a menos que se especifique lo contrario.

\section{RESUMEN DE LOS RESULTADOS}

La información sobre los efectos de restauración con técnica bulk-fill comparado con técnica incremental en cavidades clase I y clase II de Black en dientes permanentes posteriores está basada en 15 ensayos aleatorizados que incluyeron 1219 dientes.

Diez ensayos midieron el desenlace sensibilidad postoperatoria y caries secundaria (838 dientes) ${ }^{[11], ~[12], ~[13], ~[14], ~[15], ~[16], ~[17], ~[18], ~[19], ~[20], ~} 11$ ensayos evaluaron el desenlace fracaso de la restauración a mediano plazo (795 dientes) ${ }^{[8], ~[9], ~[10], ~[11], ~[12], ~[13], ~[14], ~[15], ~[16], ~[17], ~[18] ~ y ~ c u a t r o ~ e n s a y o s ~ m i d i e r o n ~ e l ~}$ desenlace fracaso de la restauración a largo plazo (386 dientes) $)^{[19], ~[20], ~[21], ~}$ [22].

El resumen de los resultados es el siguiente:

- La técnica bulk-fill podría aumentar levemente el fracaso de la restauración a mediano plazo (certeza de la evidencia baja).

- La técnica bulk-fill podría resultar en poca o nula diferencia en el fracaso de la restauración a largo plazo (certeza de la evidencia baja).

- La técnica bulk-fill probablemente resulta en poca o nula diferencia en la sensibilidad postoperatoria (certeza de la evidencia moderada).

- La técnica bulk-fill podría resultar en poca o nula diferencia en el desarrollo de caries secundaria (certeza de la evidencia baja). 


\section{Técnica bulk-fill comparado con técnica incremental para restauraciones en dientes permanentes posteriores}

\begin{tabular}{|c|c|c|c|c|}
\hline $\begin{array}{l}\text { Pacientes } \\
\text { Intervención } \\
\text { Comparación }\end{array}$ & $\begin{array}{l}\text { Dientes permaner } \\
\text { Restauración bulk } \\
\text { Restauración con }\end{array}$ & $\begin{array}{l}\text { steriores } \\
\text { incremental }\end{array}$ & & \\
\hline \multirow{3}{*}{ Desenlaces } & \multicolumn{2}{|c|}{ Efecto absoluto* } & \multirow{3}{*}{$\begin{array}{l}\text { Efecto } \\
\text { relativo } \\
\text { (IC 95\%) }\end{array}$} & \multirow{3}{*}{$\begin{array}{c}\text { Certeza de la } \\
\text { evidencia } \\
\text { (GRADE) }\end{array}$} \\
\hline & $\begin{array}{c}\text { CON } \\
\text { técnica } \\
\text { incremental } \\
\end{array}$ & $\begin{array}{l}\text { CON } \\
\text { técnica bulk-fill }\end{array}$ & & \\
\hline & \multicolumn{2}{|c|}{ Diferencia: dientes por 1000} & & \\
\hline \multirow{2}{*}{$\begin{array}{l}\text { Fracaso de la } \\
\text { restauración a } \\
\text { mediano plazo** }\end{array}$} & 56 por 1000 & 86 por 1000 & \multirow{2}{*}{$\begin{array}{l}\text { RR } 1,52 \\
(0,96 \text { a } \\
2,48)\end{array}$} & \multirow{2}{*}{$\begin{array}{c}\oplus \oplus \mathrm{Baja}^{1,2} \\
\text { Baja }\end{array}$} \\
\hline & \multicolumn{2}{|c|}{$\begin{array}{l}\text { Diferencia: } 30 \text { dientes más } \\
\text { (Margen de error: } 2 \text { menos a } 83 \text { más) }\end{array}$} & & \\
\hline \multirow[b]{2}{*}{$\begin{array}{l}\text { Fracaso de la } \\
\text { restauración a largo } \\
\text { plazo** }\end{array}$} & 72 por 1000 & 74 por 1000 & \multirow[b]{2}{*}{$\begin{array}{l}\text { RR } 1,03 \\
(0,50 \mathrm{a} \\
2,10)\end{array}$} & \multirow[b]{2}{*}{$\begin{array}{c}\oplus \oplus \bigcirc \bigcirc^{1,2} \\
\mathrm{Baja}\end{array}$} \\
\hline & \multicolumn{2}{|c|}{$\begin{array}{l}\text { Diferencia: } 2 \text { dientes más } \\
\text { (Margen de error: } 36 \text { menos a } 79 \text { más) }\end{array}$} & & \\
\hline \multirow{2}{*}{$\begin{array}{l}\text { Sensibilidad } \\
\text { postoperatoria }\end{array}$} & 0 por 1000 & 1 por 1000 & \multirow{2}{*}{$\begin{array}{l}\text { RR } 3,86 \\
(0,64 a \\
23,16)\end{array}$} & \multirow{2}{*}{$\stackrel{\oplus \oplus \oplus \bigcirc^{1}}{\text { Moderada }}$} \\
\hline & \multicolumn{2}{|c|}{$\begin{array}{l}\text { Diferencia: } 1 \text { diente más } \\
\text { (Margen de error: } 0 \text { menos a } 5 \text { más) }\end{array}$} & & \\
\hline \multirow{2}{*}{ Caries secundaria } & 5 por 1000 & 14 por 1000 & \multirow{2}{*}{$\begin{array}{l}\text { RR } 2,86 \\
(0,75 a \\
10,90)\end{array}$} & \multirow{2}{*}{$\begin{array}{c}\oplus \oplus \bigcirc \bigcirc^{1,2} \\
\text { Baja }\end{array}$} \\
\hline & \multicolumn{2}{|c|}{$\begin{array}{l}\text { Diferencia: } 9 \text { dientes más } \\
\text { (Margen de error: } 1 \text { menos a } 47 \text { más) }\end{array}$} & & \\
\hline
\end{tabular}

Margen de error: Intervalo de confianza del 95\% (IC 95\%).

RR: Riesgo relativo.

GRADE: Grados de evidencia del GRADE Working Group (ver más adelante).

*Los riesgos SIN bulk-fill están basados en los riesgos del grupo control en los estudios. El riesgo CON bulk-fill (y su margen de error) está calculado a partir del efecto relativo (y su margen de error).

** Fracaso de la restauración definido como calificación charlie o delta según criterios Ryge o USPHS modificado Se consideró mediano plazo como fracaso de la restauración antes de 5 años ( 1 a 4 años de seguimiento) y largo plazo después de 5 años (5 a 10 años de seguimiento).

${ }^{1}$ Se disminuyó un nivel de certeza de evidencia por riesgo de sesgo, ya que se encontraron limitaciones asociadas a la generación de la secuencia de aleatorización, enmascaramiento de los participantes y sesgo de realización, puesto que no se puede descartar un efecto proveniente de cointervenciones (uso de distintos adhesivos en cada grupo).

${ }^{2}$ Se disminuyó un nivel de certeza de evidencia por imprecisión, ya que a cada extremo del intervalo de confianza se tomarían decisiones diferentes.

Siga el enlace para acceder a la versión interactiva de esta tabla (Interactive Summary of Findings - iSoF)

\begin{tabular}{|l|}
\hline Acerca de la certeza de la evidencia (GRADE)* \\
\hline$\oplus \oplus \oplus \oplus$ \\
Alta: La investigación entrega una muy buena indicación del efecto probable. La probabilidad de \\
que el efecto sea sustancialmente distinto† es baja. \\
$\oplus \oplus \oplus \bigcirc$ \\
Moderada: La investigación entrega una buena indicación del efecto probable. La probabilidad de \\
que el efecto sea sustancialmente distinto† es moderada. \\
$\oplus \oplus \bigcirc \bigcirc$ \\
Baja: La investigación entrega alguna indicación del efecto probable. Sin embargo, la probabilidad \\
de que el efecto sea sustancialmente distinto† es alta. \\
$\oplus \bigcirc \bigcirc \bigcirc$ \\
Muy baja: La investigación no entrega una estimación confiable del efecto probable. La \\
probabilidad de que el efecto sea sustancialmente distinto† es muy alta. \\
\hline *Esto es también denominado 'calidad de la evidencia' o 'confianza en los estimadores del efecto'. \\
$\dagger$ Sustancialmente distinto = una diferencia suficientemente grande como para afectar la decisión. \\
\hline
\end{tabular}




\section{OTRAS CONSIDERACIONES PARA LA TOMA DE DECISIÓN}

\section{A quién se aplica y a quién no se aplica esta evidencia}

- Los resultados de este resumen son aplicables a las restauraciones clase I y clase II de Black realizadas en dientes posteriores permanentes.

- Si bien no se analizaron estudios en dientes primarios, es muy posible que la información y conclusiones reportadas por esta revisión serían extrapolables a dentición temporal y mixta.

\section{Sobre los desenlaces incluidos en este resumen}

- Los desenlaces seleccionados son aquellos considerados críticos para la toma de decisión de acuerdo a la opinión de los autores de este resumen, coincidiendo en general con los evaluados por las revisiones sistemáticas identificadas.

\section{Balance daño/beneficio y certeza de la evidencia}

- La evidencia muestra poca o nula diferencia entre usar técnica bulk-fill o incremental en el fracaso clínico a largo plazo, desarrollo de sensibilidad postoperatoria y la aparición de caries secundarias entre las restauraciones.

- Acerca del fracaso clínico a mediano plazo, la evidencia muestra que podría haber un leve perjuicio al utilizar la técnica bulk-fill en comparación a la técnica incremental, pero la certeza de la evidencia es baja.

- Considerando lo anterior, el balance entre daños y beneficios podría estar a favor de ambas intervenciones, por lo tanto deben considerarse otros aspectos para orientar la toma de decisiones como el menor tiempo clínico utilizado en una técnica bulk-fill y valores y preferencias de los pacientes y tratantes.

\section{Consideraciones de recursos}

- Ninguna de las revisiones sistemáticas ni estudios identificados realizó un análisis de costos entre la técnica bulk-fill y técnica incremental.

- Considerando las conclusiones obtenidas y la potencial ventaja de la técnica bulk-fill en términos del tiempo de trabajo requerido, es necesario realizar un análisis formal de la intervención para esclarecer su costoefectividad.

\section{Qué piensan los pacientes y sus tratantes}

- Enfrentados a la evidencia disponible, los pacientes y tratantes podrían optar por utilizar tanto la técnica incremental como la técnica bulk-fill.

- Los pacientes podrían inclinarse por el uso de la intervención debido a la disminución en el tiempo de atención, lo cual podría significar mayor comodidad para el usuario.

- Así mismo, los tratantes podrían inclinarse por el uso de la intervención considerando el tiempo clínico disponible y cantidad de pacientes por atender (centros de alto volumen).

\section{Diferencias entre este resumen y otras fuentes}

- Las conclusiones de este resumen concuerdan con las cinco revisiones sistemáticas identificadas [3][4][5][6][7], las cuales consideran que el desempeño clínico entre restauraciones posteriores con técnica bulk-fill e incremental sería similar.

- No se identificaron guías clínicas específicas que evalúen la pregunta de interés.

\section{¿Puede que cambie esta información en el futuro?}

- La probabilidad de que futuras investigaciones cambien las conclusiones de este resumen es moderada para sensibilidad postoperatoria y alta para el desarrollo de caries secundaria y fracaso de las restauraciones a mediano y largo plazo, debido a la incertidumbre de la evidencia existente.

- Identificamos 8 revisiones sistemáticas en curso en el International prospective register of systematic reviews (PROSPERO) del National institute for health research ${ }^{[23][24][25][26][27][28][29][30]}$ y 27 ensayos aleatorizados en curso en International Clinical Trials Registry Platform de la Organización Mundial de la Salud[31][32][33][34][35][36][37][38][39][40][4]][42][43][44][45][46][47][48][49][50][51][52][53][54][55] [56].

\section{CÓMO REALIZAMOS ESTE RESUMEN}

Mediante métodos automatizados y colaborativos recopilamos toda la evidencia relevante para la pregunta de interés y la presentamos en una matriz de evidencia.

\section{Siga el enlace para acceder a la versión interactiva:}

Restauración con técnica bulk-fill comparado con técnica incremental para dientes posteriores permanentes

\section{NOTAS}

Si con posterioridad a la publicación de este resumen se publican nuevas revisiones sistemáticas sobre este tema, en la parte superior de la matriz se mostrará un aviso de "nueva evidencia".

Este artículo es parte del proyecto síntesis de evidencia de Epistemonikos. Se elabora con una metodología preestablecida, siguiendo rigurosos estándares metodológicos y proceso de revisión por pares interno. Cada uno de estos artículos corresponde a un resumen, denominado FRISBEE (Friendly Summary of Body of Evidence using Epistemonikos), cuyo principal objetivo es sintetizar el conjunto de evidencia de una pregunta específica, en un formato amigable a los profesionales clínicos. Sus principales recursos se basan en la matriz de evidencia de Epistemonikos y análisis de resultados usando metodología GRADE. Mayores detalles de los métodos para elaborar este FRISBEE están descritos aquí (http://dx.doi.org/10.5867/ medwave.2014.06.5997)

La Fundación Epistemonikos es una organización que busca acercar la información a quienes toman decisiones en salud, mediante el uso de tecnologías. Su principal desarrollo es la base de datos Epistemonikos (www.epistemonikos.org).

\section{DECLARACIÓN DE CONFLICTOS DE INTERESES}

Los autores declaran no tener conflictos de intereses con la materia de este artículo.

\section{AGRADECIMIENTOS}

Este resumen de evidencia fue elaborado con el apoyo metodológico del Centro Evidencia UC, Facultad de Medicina, Pontificia Universidad Católica de Chile.

\section{Bibliografía}

1. Moraschini V, Ka Fai C, Monte Alto R, Oliveira Dos Santos G. Amalgam and Resin Composite Longevity of Posterior Restorations: A Systematic Review and Meta-Analysis. J Dent. 2015 Sep;43(9):1043-1050. doi: 10.1016/j.jdent.2015.06.005. Epub 2015 Jun 24. PMID: 26116767.

2. Lynch CD, Opdam NJ, Hickel R, Brunton PA, Gurgan S, Kakaboura A, Shearer AC, Vanherle G, Wilson NH; Academy of Operative Dentistry European Section. Guidance on posterior resin composites: Academy of Operative Dentistry - European Section. J Dent. 2014 Apr;42(4):377-83. doi: 10.1016/j.jdent.2014.01.009. Epub 2014 Jan 22. PMID: 24462699 3. Kruly PC, Giannini M, Pascotto RC, Tokubo LM, Suga USG, Marques ACR, Terada RSS. Meta-analysis of the clinical behavior of posterior direct resin restorations: Low polymerization shrinkage resin in comparison to methacrylate composite resin. PLoS One. 2018 Feb 21;13(2):e0191942. doi: 10.1371/journal.pone.0191942. PMID: 29466366; PMCID: PMC5842874.

4. Veloso SRM, Lemos CAA, de Moraes SLD, do Egito Vasconcelos BC, Pellizzer EP, de Melo Monteiro GQ. Clinical performance of bulk-fill and conventional resin composite restorations in posterior teeth: a systematic review and meta-analysis. Clin Oral Investig. 2019 Jan;23(1):221-233. doi: 10.1007/s00784-018-2429-7. Epub 2018 Mar 28. PMID: 29594349.

5. Cidreira Boaro LC, Pereira Lopes D, de SouzaASC, Lie Nakano E, Ayala Perez MD, Pfeifer CS, Gonçalves F. Clinical performance and chemical-physical properties of bulk fill composites resin -a systematic review and meta-analysis. Dent Mater. 2019 Oct;35(10):e249-e264. doi: 10.1016/j.dental.2019.07.007. Epub 2019 Aug 14. PMID: 31421957.

6. Schwendicke F, Göstemeyer G, Blunck U, Paris S, Hsu LY, Tu YK. Directly Placed Restorative Materials: Review and Network Meta-analysis. J Dent Res. 2016 Jun;95(6):61322. doi: 10.1177/0022034516631285. Epub 2016 Feb 24. PMID: 26912220.

7. Splieth $\mathrm{CH}$, Kanzow P, Wiegand A, Schmoeckel J, Jablonski-Momeni A. How to intervene in the caries process: proximal caries in adolescents and adults-a systematic review and meta-analysis. Clin Oral Investig. 2020 May;24(5):1623-1636. doi: 10.1007/s00784-02003201-y. Epub 2020 Apr 18. PMID: 32306093.

8. Frascino S, Fagundes TC, Silva U, Rahal V, Barboza A, Santos PH, Briso A. Randomized Prospective Clinical Trial of Class II Restorations Using Low-shrinkage Flowable Resin Composite. Oper Dent. 2020 Jan/Feb;45(1):19-29. doi: 10.2341/18-230-C. Epub 2019 Apr 29. PMID: 31034347.

9. Çelik Ç, Arhun N, Yamanel K. Clinical evaluation of resin-based composites in posterio restorations: a 3-year study. Med Princ Pract. 2014;23(5):453-9. doi: 10.1159/000364874. Epub 2014 Aug 12. PMID: 25115230; PMCID: PMC5586919.

10. Brackett WW, Browning WD, Brackett MG, Callan RS, Blalock JS. Effect of restoration size on the clinical performance of posterior "packable" resin composites over 18 months. Oper Dent. 2007 May-Jun;32(3):212-6. doi: 10.2341/06-87. PMID: 17555171.

11. Atabek D, Aktas N, Sakaryali D, Bani M. Two-year clinical performance of sonic-resin 
placement system in posterior restorations. Quintessence Int. 2017;48(9):743-751. doi: 10.3290/j.qi.a38855. PMID: 28849804.

12.Bayraktar Y, Ercan E, Hamidi MM, Çolak H. One-year clinical evaluation of different types of bulk-fill composites. J Investig Clin Dent. 2017 May;8(2). doi: 10.1111/jicd.12210. Epub 2016 Jan 22. PMID: 26800647.

13. Colak H, Tokay U, Uzgur R, Hamidi MM, Ercan E. A prospective, randomized, doubleblind clinical trial of one nano-hybrid and one high-viscosity bulk-fill composite restorative systems in class II cavities: 12 months results. Niger J Clin Pract. 2017 Jul;20(7):822-831. doi: 10.4103/1119-3077.212449. PMID: 28791976.

14. Arhun N, Celik C, Yamanel K. Clinical evaluation of resin-based composites in posterior restorations: two-year results. Oper Dent. 2010 Jul-Aug;35(4):397-404. doi: 10.2341/09-345C. PMID: 20672723

15. Alkurdi RM, Abboud SA. Clinical evaluation of class II composite: Resin restorations placed by two different bulk-fill techniques. J Orofac Sci. 2016 May;8(1):34-9. doi: 10.4103/0975-8844.181926.

16. Karaman E, Keskin B, Inan U. Three-year clinical evaluation of class II posterior composite restorations placed with different techniques and flowable composite linings in endodontically treated teeth. Clin Oral Investig. 2017 Mar;21(2):709-716. doi: 10.1007/s00784-016-1940-y. Epub 2016 Aug 19. PMID: 27538739.

17. Manhart J, Chen HY, Hickel R. Clinical evaluation of the posterior composite Quixfil in class I and II cavities: 4-year follow-up of a randomized controlled trial. J Adhes Dent. 2010 Jun;12(3):237-43. doi: 10.3290/j.jad.a17551. PMID: 20157663.

18. Yazici AR, Antonson SA, Kutuk ZB, Ergin E. Thirty-Six-Month Clinical Comparison of Bulk Fill and Nanofill Composite Restorations. Oper Dent. 2017 Sep/Oct;42(5):478-485. doi: 10.2341/16-220-C. Epub 2017 Jun 5. PMID: 28581919

19. van Dijken JW, Pallesen U. Posterior bulk-filled resin composite restorations: A 5-year randomized controlled clinical study. J Dent. 2016 Aug;51:29-35. doi: 10.1016/j. jdent.2016.05.008. Epub 2016 May 26. PMID: 27238052.

20. van Dijken JWV, Pallesen U. Bulk-filled posterior resin restorations based on stressdecreasing resin technology: a randomized, controlled 6-year evaluation. Eur J Oral Sci. 2017 Aug;125(4):303-309. doi: 10.1111/eos.12351. Epub 2017 May 19. PMID: 28524243. 21. Fagundes TC, Barata TJ, Carvalho CA, Franco EB, van Dijken JW, Navarro MF. Clinical evaluation of two packable posterior composites: a five-year follow-up. J Am Dent Assoc. 2009 Apr;140(4):447-54. doi: 10.14219/jada.archive.2009.0194. PMID: 19339534

22. Heck K, Manhart J, Hickel R, Diegritz C. Clinical evaluation of the bulk fill composite QuiXfil in molar class I and II cavities: 10-year results of a RCT. Dent Mater. 2018 Jun;34(6):e138-e147. doi: 10.1016/j.dental.2018.03.023. Epub 2018Apr 7. PMID: 29636239 23. Abreu N. Analysis of the longevity of composite resins Ormocer, Bulk Fill in posterior restorations: a systematic review. PROSPERO, 2019. ID: CRD42019134990. Available from: https://www.crd.york.ac.uk/prospero/display_record.php?ID=CRD42019134990

24. Meereis C, Suárez C, Rosa W, Almeida S, Silva A, Piva E. Bulk fill resin composite: a systematic review and meta-analysis of in-vitro and clinical studies. PROSPERO, 2017. ID: CRD42017064424. Available from:

https://www.crd.york.ac.uk/prospero/display record.php?ID=CRD42017064424

25. Giubilei F, Souza C, Silva C, Alencar C, Esteves R. Bulk-fill resin vs conventional resins on post-operative sensivity: A systematic review . PROSPERO, 2020. ID: CRD42020183793. Available from:

https://www.crd.york.ac.uk/prospero/display record.php?ID=CRD42020183793

26. Soares A, dos Santos L, Coelho M, Macedo L. Clinical failure rate of restorations with bulk fill composite resin in primary and permanent posterior teeth: a systematic review. PROSPERO, 2017. ID: CRD42017064063. Available from: https://www.crd.york.ac.uk/ prospero/display record.php?|D=CRD42017064063

27. Mamede S, Queiroz de Melo G, Fonseca O. Do bulk fill resins composite show clinical performance comparable to conventional resins composite in direct posterior restorations? PROSPERO, 2016. ID: CRD42016053436. Available from:

https://www.crd.york.ac.uk/prospero/display_record.php?ID=CRD42016053436

28. Kunz P, Castiglia C, Wambier L, Correr G, Schaia J, Cunha L. Does the bulk fill resin composite have higher clinical performance than incremental resin in posterior teeth? A systematic review. PROSPERO, 2018. ID: CRD42018108450. Available from: https://www.crd.york.ac.uk/prospero/display_record.php?ID=CRD42018108450 29. Dantas M, Soares F. Does the use of a bulk-fill resin composite really decrease the clinical time or the number of increments in restorations in posterior teeth?. PROSPERO, 2018. ID: CRD42018084149. Available from:

https://www.crd.york.ac.uk/PROSPERO/display_record.php?RecordID=84149

30. Gomes G, Reis A, Loguercio A, Bakaus T, Gruber Y. Impact of bulk vs layering technique for posterior restoration on postoperative sensitivity: a systematic review. PROSPERO, 2016. ID: CRD42016036771. Available from:

https://www.crd.york.ac.uk/prospero/display_record.php?ID=CRD42016036771

31. Chen Z. The clinical evaluation of the restoration of the bulkfil flowable and conventional composite resin after root canal treatment. International Clinical Trials Registry Platform de la Organización Mundial de la Salud. 2017. ID: ChiCTR-IOR-15006410. Available from: https://apps.who.int/trialsearch/Trial2.aspx?TriallD=ChiCTR-IOR-15006410

32. Tawfik M. One-year Clinical Evaluation of Bulk-fill Versus Conventional Incremental Posterior Restoration by FDI. International Clinical Trials Registry Platform de la Organización Mundial de la Salud. 2016. ID: NCT02891967. Available from:

https://apps.who.int/trialsearch/Trial2.aspx?TriallD=NCT02891967

33. Peters M. Investigation OfABulk Fill Composite In Class II Restorations In Adult Subjects. International Clinical Trials Registry Platform de la Organización Mundial de la Salud. 2017 ID: NCT03175627. Available from:

https://apps.who.int/trialsearch/Trial2.aspx?TriallD=NCT03175627

34. Barceleiro M. Clinical Evaluation of Bulk Fill Composite Restorations Associated to Universal Adhesive System. International Clinical Trials Registry Platform de la Organización Mundial de la Salud. 2017. ID: NCT03343184. Available from:

https://apps.who.int/trialsearch/Trial2.aspx?TriallD=NCT03343184

35. Sakkir N. Clinical Evaluation of Bulk-fill vs Layered Composite Resin in Class I and II Posterior Restorations: A Randomized Controlled Pilot Study. International Clinical Trials Registry Platform de la Organización Mundial de la Salud. 2017. ID: NCT03306602. Available from: https://apps.who.int/trialsearch/Trial2.aspx?TriallD=NCT03306602
36. Yacizi A. A 24-month Clinical Evaluation of Different Bulk-fill Restorative Resins in Class II Restorations. International Clinical Trials Registry Platform de la Organización Mundial de la Salud. 2018. ID: NCT03527953. Available from:

https://apps.who.int/trialsearch/Trial2.aspx?TriallD=NCT03527953

37. Ali R. Evaluation of Post-operative Sensitivity of Bulk Fill Resin Composite Versus the Nano Resin Composite. International Clinical Trials Registry Platform de la Organización Mundial de la Salud. 2019. ID: NCT03792178. Available from:

https://apps.who.int/trialsearch/Trial2.aspx?TriallD=NCT03792178

38. Vildosola P. Clinical Performance of Bulk-fill Resins Composite Restorations on Posterio Tooth of Adults Patients. Randomized Doble-blind Trial. International Clinical Trials Registry Platform de la Organización Mundial de la Salud. 2017. ID: NCT03230604. Available from: https://apps.who.int/trialsearch/Trial2.aspx?TriallD=NCT03230604

39. Queiroz G. Class I restoration performance with bulk fill composites randomized clinical trial. International Clinical Trials Registry Platform de la Organización Mundial de la Salud. 2015. ID: RBR-5v6dsj. Available from:

https://apps.who.int/trialsearch/Trial2.aspx?TriallD=RBR-5v6ds

40. Wang X. A Randomized Multicenter Two-Arm Clinical Study to Evaluate the Safety and Efficacy of Filtek ${ }^{\mathrm{TM}}$ Bulk Fill Posterior Restorative in Class I and II Restorations. International Clinical Trials Registry Platform de la Organización Mundial de la Salud. 2018 ID: NCT03764059. Available from:

https://apps.who.int/trialsearch/Trial2.aspx?TriallD=NCT03764059

41. Ferraz T. Longitudinal Clinical Assessment of Class II Cavity Restorations Using Bulk-Fill Technique: A Randomized Clinical Study. International Clinical Trials Registry Platform de la Organización Mundial de la Salud. 2018. ID: RBR-9z6hx2. Available from:

https://apps.who.int/trialsearch/Trial2.aspx?TriallD=RBR-9z6hx2

42. Rocha $\mathrm{C}$. Evaluation of admira fusion vs admira fusion xtra resins in posterior teeth restorations: randomized double-blind clinical trial. International Clinical Trials Registry Platform de la Organización Mundial de la Salud. 2018. ID: RBR-989h4f. Available from: https://apps.who.int/trialsearch/Trial2.aspx?TriallD=RBR-989h4f

43. Rocha C. Clinical evaluation of restorations in posterior teeth using bulk fill ormoce composite - a randomized clinical trial. International Clinical Trials Registry Platform de la Organización Mundial de la Salud. 2019. ID: RBR-6mvp9w. Available from:

https://apps.who.int/trialsearch/Trial2.aspx?TriallD=RBR-6mvp9w

44. Coelho M, Linares S. Clinical evaluation of resin restorations of posterior teeth performed at the são luis dental specialty Center - randomized clinical trial. International Clinical Trials Registry Platform de la Organización Mundial de la Salud. 2020. ID: RBR-7hbmjp Available from: https://apps.who.int/trialsearch/Trial2.aspx?TriallD=RBR-7hbmjp

45. Batra R. Comparative Evaluation Of Cention â??N, Equia Forte And Activa Bulk Fill Material In Class I Posterior Restortions- A Randomized Controlled Trial. International Clinical Trials Registry Platform de la Organización Mundial de la Salud. 2018. ID: CTRI/2018/10/015880. Available from: https://apps.who.int/trialsearch/Trial2.aspx?TriallD= CTRI/2018/10/015880

46. Elahassan M. Clinical evaluation of direct resin composite restorations of endodontically treated molars using dual cured self-adhesive system with bulk fill composite versus incremental packing technique. International Clinical Trials Registry Platform de la Organización Mundial de la Salud. 2016. ID: PACTR201602001466307. Available from: https://apps.who.int/trialsearch/Trial2.aspx?TriallD=PACTR201602001466307

47. Aragão B. Bulk fill composite resins: randomized and double-blind clinical trial. International Clinical Trials Registry Platform de la Organización Mundial de la Salud. 2018 ID: RBR-8fdnky. Available from: https://apps.who.int/trialsearch/Trial2.aspx?TriallD=RBR8fdnky

48. 3M. Clinical Performance of Incremental and Bulk Fill Composites in Class II Restorations. International Clinical Trials Registry Platform de la Organización Mundial de la Salud. 2016. ID: NCT02889835. Available from:

https://apps.who.int/trialsearch/Trial2.aspx?TriallD=NCT02889835

49. Sirin E. Twenty-Four-Month Clinical Comparison of Two Bulk-Fill and Microhybrid Composite Restorations. International Clinical Trials Registry Platform de la Organización Mundial de la Salud. 2020. ID: NCT04306549. Available from:

https://apps. who.int/trialsearch/Trial2.aspx?TriallD=NCT04306549

50. Yazici A. Clinical Comparison of Bulk-fill Restorative and Nano-fill Resin Composite. International Clinical Trials Registry Platform de la Organización Mundial de la Salud. 2020 ID: NCT04320576. Available from: https://clinicaltrials.gov/ct2/show/record/NCT04320576

51. Jolayemi J. Clinical Evaluation of Incremental and Bulk-Fill Composite Restorative Techniques on Enlarged Posterior Occlusal and Proximal Carious Cavities in Nigerian Adults. International Clinical Trials Registry Platform de la Organización Mundial de la Salud. 2019. ID: PACTR202001541490698. Available from:

https://apps.who.int/trialsearch/Trial2.aspx?TriallD=PACTR202001541490698

52. Kumar A. To evaluate postoperative sensitivity in four different tooth colored materials in patients. International Clinical Trials Registry Platform de la Organización Mundial de la Salud. 2019. ID: CTRI/2019/10/021552. Available from:

https://apps.who.int/trialsearch/Trial2.aspx?TriallD=CTRI/2019/10/021552

53. Frese C. Randomized controlled clinical trial evaluating Filtek Bulk Fill Posterior Restorative in a split-mouth study design. International Clinical Trials Registry Platform de la Organización Mundial de la Salud. 2020. ID: DRKS00013799. Available from: https://apps. who.int/trialsearch/Trial2.aspx?TriallD=DRKS00013799

54. Araujo D. Clinical evaluation of tooth restoration using an agent by only one insertion International Clinical Trials Registry Platform de la Organización Mundial de la Salud. 2020. ID: RBR-2h9qkd. Available from:

https://apps.who.int/trialsearch/Trial2.aspx?TriallD=RBR-2h9qkd

55. Balkaya H. Two-Year Clinical Comparison of Three Different Restorative Materials in Class II Cavities. International Clinical Trials Registry Platform de la Organización Mundial de la Salud. 2019. ID: TCTR20190405001. Available from:

https://apps.who.int/trialsearch/Trial2.aspx?TrialID=TCTR20190405001

56. Walter R. Clinical Investigation of a New Bulk Fill Composite Resin in the Restoration of Posterior Teeth. International Clinical Trials Registry Platform de la Organización Mundial de la Salud. 2015. ID: NCT02572570. Available from:

https://apps.who.int/trialsearch/Trial2.aspx?TriallD=NCT02572570 